\title{
Current treatment strategy in the management of vestibular schwannoma
}

\author{
Basant K Misra, Harshad R Purandare, Rahul S Ved, Anshul A Bagdia, Pandurang B Mare
}

Department of Neurosurgery and Gamma Knife Surgery, P D Hinduja National Hospital and Medical Research Centre, VS Marg, Mahim, Mumbai-400 016, India

\author{
Address for correspondence: \\ Dr. Basant K Misra, \\ Department of Neurosurgery and \\ Gamma Knife Surgery, P D Hinduja \\ National Hospital and Medical \\ Research Centre, VS Marg, Mahim, \\ Mumbai-400 016, India. \\ E-mail: basantkmisra@gmail.com
}

DOI: $10.4103 / 0028-3886.53263$

\begin{abstract}
Background: The changing trends in the management of vestibular schwannoma (VS) in our practice over the last two decades as well as the current status are presented here. Materials and Methods: The observations are based on the experience of 559 consecutive cases of VS operated by the first author between 1987 and 2008, 438 of which were operated by microsurgery and 139 by gamma knife radiosurgery (GKR) ( 18 of which were previously operated by the authors). A detailed analysis of microsurgically managed patients in two different periods ( 100 consecutive patients each before 1993 and 2008) were compared to see the changing trend and document current results. Results and Discussion: In the initial experience (1990s), the emphasis in microsurgery was preserving life, total excision of tumor and preservation of function in that order. In the $2 I^{\text {st }}$ century, the emphasis in microsurgery has been all about functional preservation. In 100 consecutive cases of VS (excluding neurofibromatosis-2) that were treated microsurgically between 2005-08, there were four small tumors $(<2 \mathrm{~cm}), 14$ medium-sized tumors $(2-3$ $\mathrm{cm})$ and 82 large tumors $(\geq 3 \mathrm{~cm})$. The total excision rate was $83 \%$. The facial nerve anatomical preservation rate was $96 \%$ and function was Grade III House-Brackmann (HB) or better in $87 \%$. Both the total excision rate and facial function of Grade II HB or better were $100 \%$ in cases with tumor size less than three $\mathrm{cm}$. Functional hearing preservation was achieved in ten cases. There was no operative mortality. Conclusion: Total excision of VS, though aimed at, is no more pursued at the cost of facial function. Moreover, microsurgery, radiosurgery and observation are all valid options in the management of VS and choosing the correct modality helps in achieving optimal outcome.
\end{abstract}

Key words: Acoustic neuroma, microsurgery, observation, radiosurgery, treatment

\section{Introduction}

Since its first successful removal, the history and progress of vestibular schwannoma (VS) management parallels that of neurosurgery..$^{[1]}$ Thus it is but natural that the present day trend of minimal invasiveness should also have a major influence in the management of a patient with VS. In fact, Cushing's recommendation of subtotal excision in VS is as old as neurosurgery itself. ${ }^{[2]}$ While the debate regarding the best microsurgical approach to a given VS continues, it has been surpassed by the debate about the role of observation, gamma knife radiosurgery (GKR) and microsurgery in the management of VS. ${ }^{[3]}$ The changes that have taken place in the management of VS in our practice over the last two decades as well as the current protocol and results are presented here.

\section{Materials and Methods}

An analysis of 909 cases of cerebellopontine angle lesions operated by the first author since 1987 yielded 559 cases of VS operated at two institutes till 2008. Microsurgery was the primary option in 438 and GKR was done in 139, of which 18 were previously operated by the authors. A prescription dose of 13 Gray (Gy) to $50 \%$ isodose

Part of the material was presented as Presidential Oration by Dr. BK Misra in NSI Annual Conference 2008 
was employed in the majority of the cases. A detailed analysis of microsurgically managed patients in two different periods (100 consecutive patients each before 1993 and 2008) were compared to see the changing trend and document current results. Though not included in these figures, there were a number of cases of VS which were only observed. Retromastoid retrosigmoid route was the microsurgical approach in all the cases. Till the early 90s, microsurgery was performed in the park bench position. In the last 15 years the patients have been operated in the supine position (barring a few) with a sandbag under the shoulder and head turned contralaterally with the sagittal plane parallel to the floor and neck flexed. Some of the key steps in the retrosigmoid approach employed by the authors were opening of the foramen magnum in large tumors, initial cerebrospinal fluid (CSF) release, minimal/no retraction of cerebellum, defining and remaining in the tumorarachnoid plane, significant decompression of tumor before dissection of the tumor capsule, six to eight $\mathrm{mm}$ drilling of internal auditory meatus (IAM) to preserve the labyrinth, excision of the lateral extent of tumor in the IAM with endoscopic assistance, sealing of the IAM by fat and fibrin glue, reinforcing by fat the primarily closed dura and replacement of the bone flap before closure of the wound in layers.

While intraoperative facial nerve monitoring was carried out in the majority of the cases, intraoperative brainstem auditory evoked response (BAER) was available only in the second phase. While a large number of patients were deaf preoperatively, there were some patients (even with large tumours) who had preserved hearing. All patients who had a preserved hearing had a preoperative BAER. Intraoperative BAER monitoring was carried out only when wave one to wave five could be detected preoperatively. It is also important to note that BAER could not be elicited (or only wave one was elicited) preoperatively in a number of patients in spite of the presence of functional hearing in audiogram. Constant irrigation while drilling IAM, no coagulation near the nerves, and side to side dissection and not in the medial to lateral or lateral to medial direction were practiced to preserve functional integrity of the cranial nerves. The patients with an uneventful postoperative period were usually given the option to be discharged on the fourth or fifth postoperative day (POD) after a contrast enhanced computed tomography (CECT) of the brain and to come back for suture removal on the eighth POD. The first follow-up was after six to eight weeks with a contrast magnetic resonance imaging (MRI) of the brain, and subsequently at six-monthly intervals (with repeat imaging at six-monthly intervals for patients with subtotal excision). The facial nerve function at the first follow-up at six to eight weeks (at discharge in patients lost to follow-up) was recorded for this tabulation.

\section{Results}

A consecutive series of 100 cases of VS treated microsurgically by the senior author was published in 1993. ${ }^{[4]}$ The changes in the treatment strategy and the results achieved were analyzed by comparing the series of 1993 to 100 consecutive microsurgically treated VS from 2005-08. The tumor size was smaller in the second group indicating earlier detection [Table 1]. In spite of a trend towards earlier detection, there were still 39\% giant tumors (four $\mathrm{cm}$ or more) in the 2008 group. The total excision rate was a high, $94 \%$ in the first phase while it was $83 \%$ in the second phase. It is important to mention that while the total excision was classified on a postoperative contrast MRI in the second phase, in the '93 series, CECT scan was more often the postoperative imaging modality. In the second phase, total excision rate was $100 \%$ in tumors less than three $\mathrm{cm}, 86 \%$ in tumors that were three to four $\mathrm{cm}$ and $72 \%$ in giant tumors (four $\mathrm{cm}$ or bigger). However, the facial function was better in the second phase with $96 \%$ facial nerve continuity in the later phase as opposed to $77 \%$ in the earlier phase.

In the last 100 cases, overall $87 \%$ patients had facial nerve function of Grade III_House and Brackmann ${ }^{[5]}(\mathrm{HB})$ or better [Table 2].

The postoperative facial function was directly related to the size of the tumor [Table 3]. Useful hearing was preserved in ten cases, six of whom had tumors larger than three $\mathrm{cm}$. One patient with a large tumor had improvement in his hearing postoperatively after a total excision [Figure 1].

\begin{tabular}{lcc}
\hline \multicolumn{3}{l}{ Table 1: Tumor size (Microsurgery) } \\
\hline Size & $1993(100)$ & $2008(100)$ \\
\hline Large $(>3 \mathrm{~cm})$ & 93 & 82 \\
Medium $(2-3 \mathrm{~cm})$ & 7 & 14 \\
Small $(<2 \mathrm{~cm})$ & 0 & 4 \\
Mortality & $1 \%$ & $0 \%$ \\
\hline
\end{tabular}

Table 2: Facial function (2008 microsurgery)

$\left.\begin{array}{llll}\hline \text { Post-op facial function } & \% & \\ \hline \text { Anatomic continuity } & 96 & \\ \text { Grade I-II (HB) } & 73 & \\ \text { Grade III (HB) } & 14 & \end{array}\right\} 87 \%$

Table 3: Facial function vis-à-vis size of tumor (2008 microsurgery)

\begin{tabular}{lcccc}
\hline Size & \multicolumn{5}{c}{ Facial function grade in \% } \\
\cline { 2 - 5 } & I & II & III & IV or worse \\
\hline Small & 100 & - & - & - \\
Medium & 93 & 7 & - & - \\
Large & 40 & 27 & 17 & 16 \\
\hline
\end{tabular}




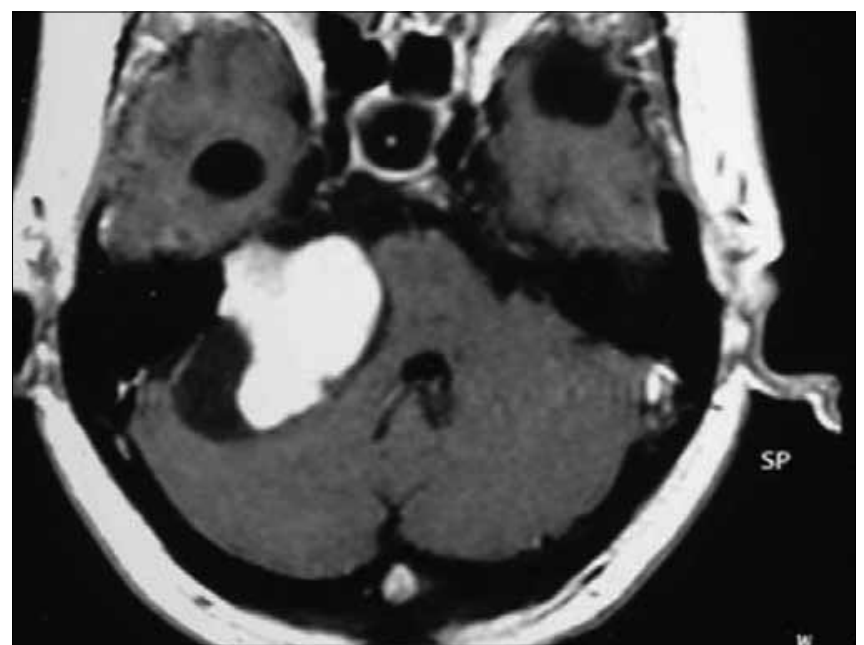

Figure 1a: Preoperative MRI (T1W CE) showing a large VS

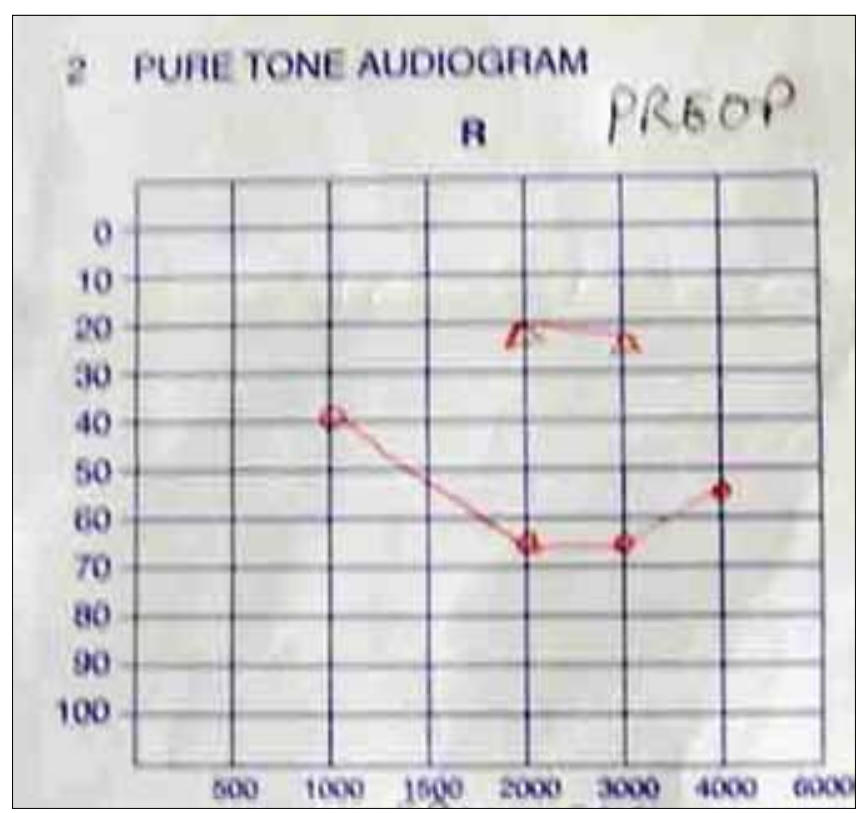

Figure 1c: Preoperative audiogram before microsurgery

The microsurgical operative mortality was $1 \%$ in the earlier series and nil recently. In fact there has been no operative mortality in the authors' practice in the last decade, the last case being one who succumbed from postoperative meningitis following CSF rhinorrhea in 1999. The commonest postoperative complication was CSF rhinorrhea [Table 4] occurring in nine patients, six of which required surgical repair. Pseudomeningocele occurred in six patients but all resolved on conservative management. Postoperative lower cranial nerve (LCN) dysfunction, the bugbear of the past, only occurred in one patient who did not have it preoperatively. The other eight patients had it preoperatively. Four patients who were discharged with a nasogastric tube or percutaneous endoscopic gastrostomy (PEG) tube had significant preoperative swallowing difficulty. Only two of the six

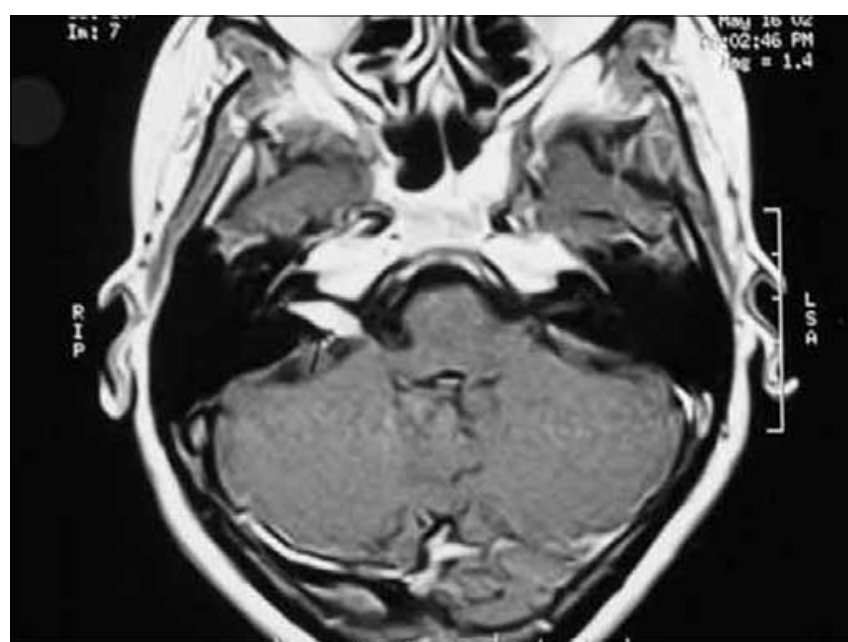

Figure 1b: Postoperative MRI (T1W CE) showing total excision (Note fat packing in the IAM)

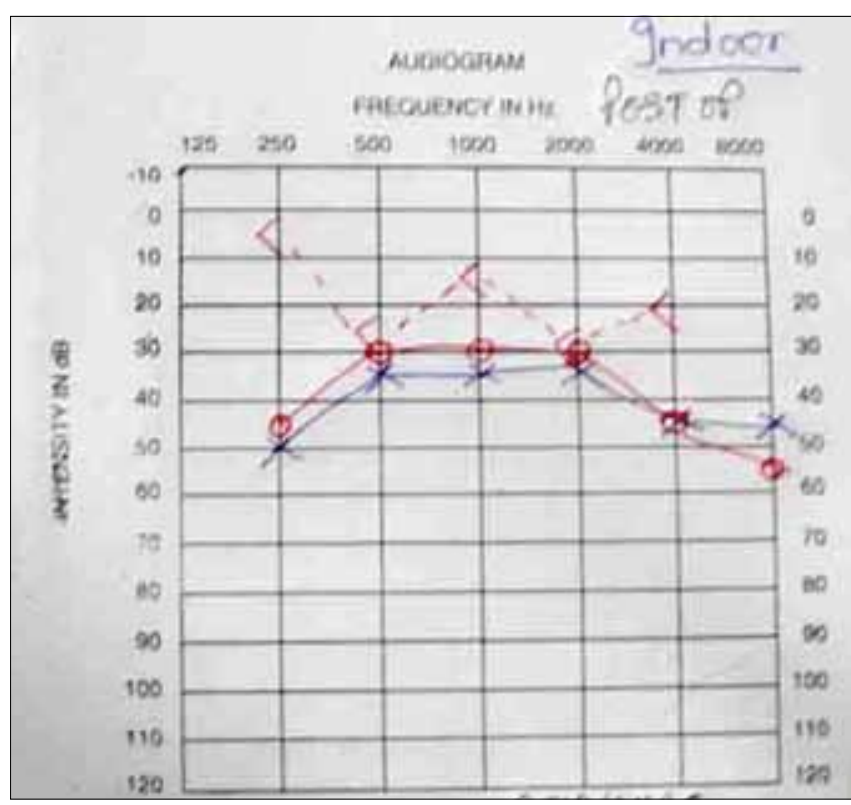

Figure 1d: Postoperative audiogram showing improvement of hearing after the excision

\begin{tabular}{lc}
\hline Table 4: Complications & \\
\hline Postoperative complications & Number of patients \\
\hline CSF rhinorrhea & 09 \\
$\quad$ Requiring surgical repair & 06 \\
Pseudomeningocele & 05 \\
Lower cranial nerve paresis & \\
$\quad$ Preoperative & 07 \\
$\quad$ Postoperative & 08 \\
Postoperative Ryle's tube feeding & \\
$\quad$ Transient & 08 \\
$\quad$ At discharge & 04 \\
Chest infection & 06 \\
Wound infection & 01 \\
Deep venous thrombosis & 00 \\
Meningitis & 00 \\
Mortality & 00 \\
\hline
\end{tabular}


patients with postoperative chest infection had normal preoperative LCN function. One patient had delayed wound infection which required surgical drainage and there was no case of meningitis. In the later series (200508), the follow-up ranged from one month to 48 months. Thirteen patients were lost to follow-up.

GKR was generally reserved by the author for tumors less than $2.5 \mathrm{~cm}$ in diameter. Of the 139 patients thus treated, three failed treatment and needed microsurgery. One patient had facial palsy from a previous operation (preGK) in another centre. In the other two, near total excision was achieved with preservation of normal facial function [Figure 2]. Two patients developed transient Grade II HB facial weakness. Detailed evaluation of their hearing status is in progress. Another complaint following GKR in some patients has been a pulling sensation in the face and some others have had paraesthesia. Symptoms of giddiness and imbalance often got worse after GK and

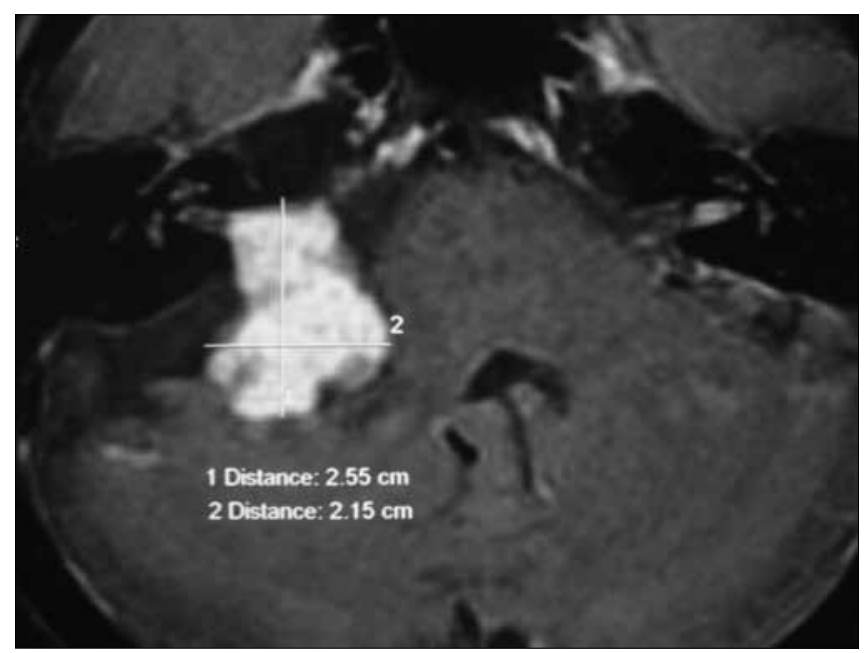

Figure 2a: CE T1W axial MRI showing a $2.5 \mathrm{~cm}$ VS

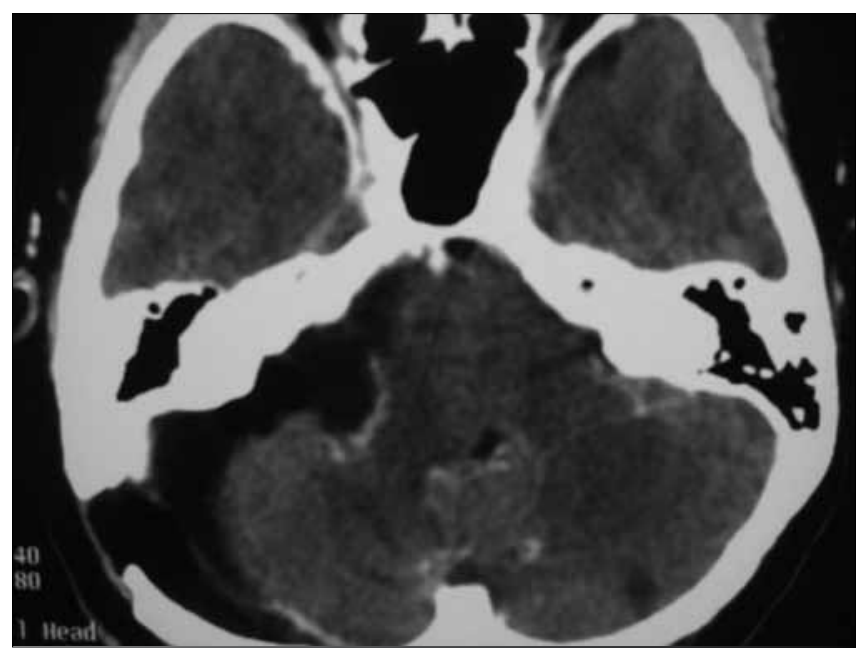

Figure 2c: Post-microsurgery CECT showing near-total excision of tumor took longer to settle down than in microsurgery.

\section{Discussion}

Sandifort of Leyden, Leveque-Lasource and Charles Bell are widely credited with the first autopsy description, first clinico-pathological correlation and first clinical description of VS respectively. ${ }^{[2,6]}$ While Balance of London is commonly credited with the first successful surgery in VS in 1892, Cushing credits Annandale of Edinburgh with the first successful surgery in 1895. ${ }^{[2]}$ Seminal papers on the clinical presentation by Cushing and neurological manifestation by House have laid the foundation for the early diagnosis of VS. ${ }^{[2,7]}$

Atypical presentation of VS is not rare, however, as has been highlighted in a classic paper by Shephard and Wadia. ${ }^{[8]}$ Though Cushing was the pioneer in achieving a significant successful operative series of VS, Dandy's

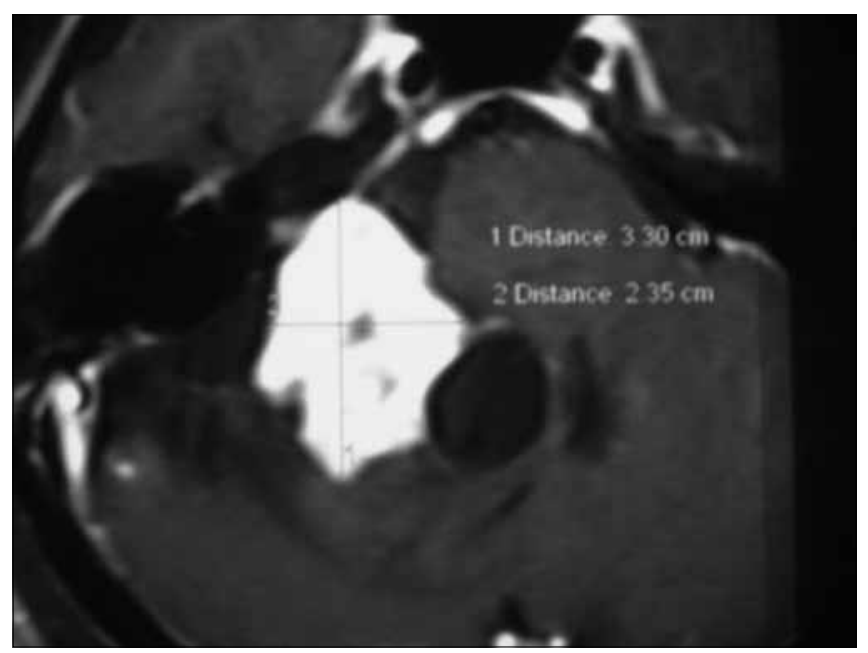

Figure 2b: Post-GK follow-up CE MRI demonstrating significant increase in tumor size and perilesional edema

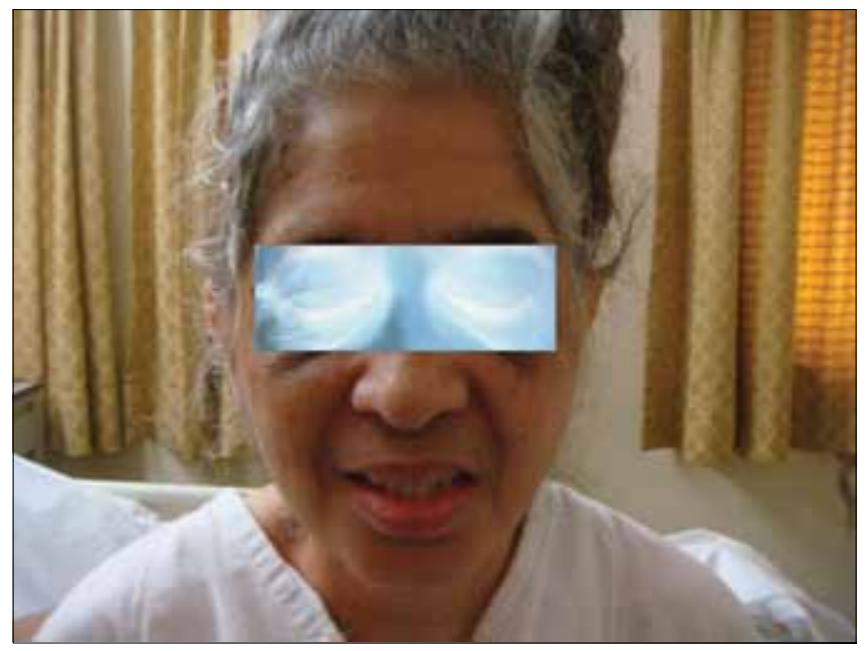

Figure 2d: Normal facial function 
outstanding results after total excision of VS were equally important in paving the way for many neurosurgeons to conquer what Cushing named "the bloody angle, the gloomy corner of neurological surgery". ${ }^{[2,9]}$ Noteworthy contributions in refining microsurgical excision of VS have been made by House, Malis, Yasargil and Samii over the years. ${ }^{[6,10-12]}$ Lars Leksell, the founder of GKR and Ladislav Steiner from Sweden have established and the Pittsburgh group popularized the role of radiosurgery as an established management protocol in VS. ${ }^{[13,14]}$

\section{Microsurgery}

In the microsurgical era, three different surgical approaches, retrosigmoid (RS), translabyrinthine (TL) and the middle cranial fossa (MCF) are commonly utilized. All the patients in the authors series have, however, been operated through the retrosigmoid route as this is the only route that is not only adequate for all sizes of tumor but also suitable for preservation of facial function and hearing. No doubt enough literature is available now to document TL and MCF approaches as excellent means of tackling selected tumors. ${ }^{[15]}$ Though the authors have used both these approaches extensively for other skull base lesions, it has not been necessary to change the strategy of retrosigmoid route for VS over the years. ${ }^{[16-21]}$ The authors' indication for microsurgery as the first choice today are

- All tumors $>2.5 \mathrm{~cm}$ in the largest diameter.

- Any patient with brainstem dysfunction, significant mass effect, raised intracranial pressure (ICP) or rapidly progressing symptomatology.

- Young patient (<50 years) with any tumor size.

Though there have been some changes in the technique employed, the basic retromastoid retrosigmoid approach has not changed over the years. ${ }^{[4,16-21]}$ Presently, the author employs a supine position with head turned to the contralateral side (with a sandbag under the ipsilateral shoulder) as opposed to the lateral position, and a craniotomy as opposed to craniectomy earlier.

The facial nerve preservation rate was a high $96 \%$ in 2008 as compared to $77 \%$ in 1993. A tradeoff has been a lower total excision rate ( $83 \%$ compared to $94 \%$ in 1993) because of the recent emphasis on good facial function. Hence a thin sliver of tumor left over the facial nerve, which showed up as enhancing tumor on postoperative MRI, was accepted as better for the patient though classified as subtotal excision. The authors are well aware of the problem of poor follow-up in our country and the risk of subtotal excision. Another problem is the inability of the patient to go for radiosurgery for a subtotally excised tumor because of the low socioeconomic status. Hence proper preoperative counseling and individualizing the goal of surgery in a given patient will go a long way to protect the patient's interest. However, it is also important to remember that a thin sliver of tumor left attached to the nerves infrequently grows to produce symptoms and even if it grows, it can be operated again with excellent results and hence the emphasis on functional preservation. Though hearing preservation after microsurgery is unpredictable, especially in large tumors, six patients with large tumors had useful hearing postoperatively, one of whom in fact had improvement in his hearing. Very few authors have been able to achieve that. ${ }^{[12,22]}$

\section{Radiosurgery}

More and more cases of VS are being treated by radiosurgery the world over, especially as patients are presenting earlier with smaller tumors. Minimal hospitalization, no general anesthesia and avoiding an open surgery are particularly appealing to the patient. Reports of long-term control with good cranial nerve function has led to increased enthusiasm for its use by the physician. ${ }^{[23-26]}$ Earlier reports of increased cranial nerve dysfunction have reduced significantly with reduction of tumor dose. The concern, however, is long-term control with the lower dosage. The other major concern has been the risk of malignant degeneration following radiosurgery. However, to date, there is no proof to suggest any realistic risk of malignancy following GKR. ${ }^{[27]}$ Presently 12-14 Gray (Gy) is prescribed to 50\% isodose in GKR. The reported long-term control rate is between $93 \%$ and $97 \%$. Though, there have been recent reports of even improved hearing after GKR, preservation is unpredictable. ${ }^{[28-30]}$ Today our indications for GKR are patients with tumors of $2.5 \mathrm{~cm}$ or less in maximum diameter and

- high medical risk

- elderly patients or

- recurrent or residual tumor showing growth.

The best modality of treatment for VS less than three cm, especially in young patients, is still controversial. . $3,19,29,31]$ There is no Class one evidence till date to recommend one modality over the other. While post-procedure morbidity is generally less and cranial nerve function is better with radiosurgery, the control/eradication rate is better with microsurgery. ${ }^{[3,29]}$ Long-term data of tumor control with radiosurgery is still not available. In a recent study of small VS comparing functional outcomes following radiosurgery and microsurgery, Coelho et al., concluded that microsurgery was better than radiosurgery in imbalance control and resulted in minimal morbidity comparable to radiosurgery. ${ }^{[31]}$

Even today, the authors advocate microsurgery as the first choice in young patients with tumors less than $2.5 \mathrm{~cm}$ as our results in this group are excellent with 
$100 \%$ preservation of facial function and eradication of tumor for good. Our present management protocol for extracanalicular VS is presented in Table 5.

\section{Observation}

Studies on the natural history of VS have shown that there are some tumors which do not grow and remain static over a long period of time. ${ }^{[32,33]}$ Intracanalicular acoustic neuroma (ICAN), small tumors and tumors picked up incidentally, thus could merit a period of follow-up. Due to the low socioeconomic status of many patients and poor follow-up in our country, one is weary of advising observation except in ICAN. Though the numbers are not included in this series, the authors have advised observation in some patients mainly elderly, those with minimal or only auditory/vestibular dysfunction and those with incidentally picked up tumors; provided there is no doubt about follow-up of such patients.

Special situations (SS): Some special clinical settings need particular mention. Our protocols in such situations are as follows:-

\section{SS 1: VS in a pregnant lady}

(i) Patients with small tumors are allowed to carry on through pregnancy and normal delivery.

(ii) Patients with tumors less than three $\mathrm{cm}$ but minimally symptomatic are advised to continue pregnancy but advised elective Caesarean section delivery.

(iii) Symptomatic patients with large tumors are advised microsurgery, preferably after the first trimester. Quick subtotal excision is performed to provide maximum safety to both mother and child. The

Table 5: Vestibular schwannoma - Treatment algorithm

Extracanalicular vestibular schwannoma

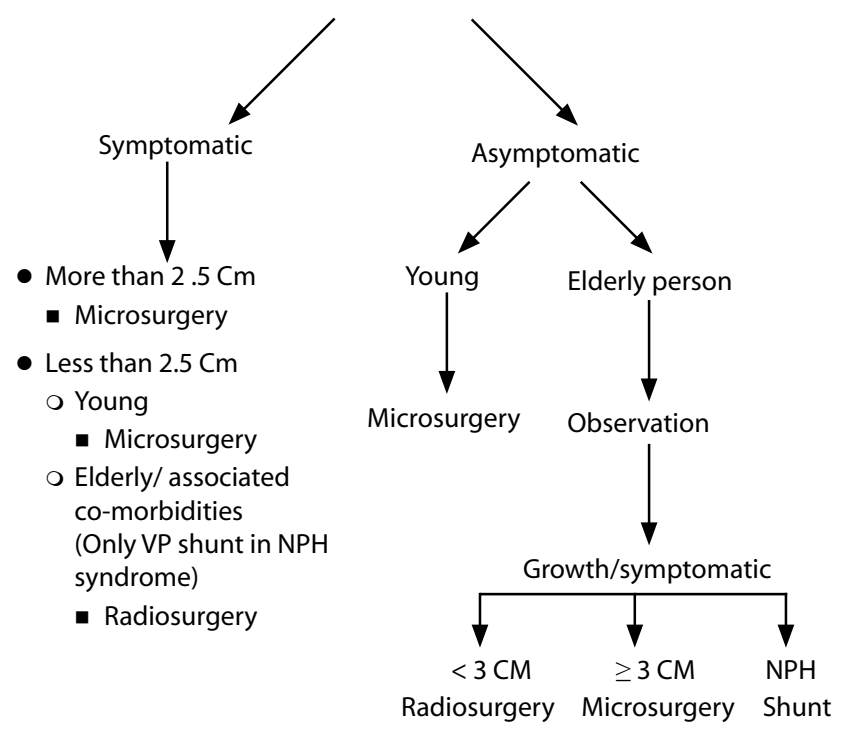

tumor is usually much more vascular in pregnancy. The patient can then go for normal pregnancy and delivery. The residual tumor, if significant, can then be excised at a later date.

\section{SS 2: Cystic/Hemorrhagic tumor}

Patients harboring cystic tumors and those presenting with macro hemorrhages should be treated by microsurgery. Large-size, Antoni B type tumors and telangiectasia pattern in the tumor make it more prone to both cystic and hemorrhagic transformation. ${ }^{16]}$ These tumors have a friable capsule leading to greater dissection difficulty. However, no effort should be spared for total excision as these have a higher risk of recurrence and postoperative hemorrhage.

\section{SS 3: VS with malignant edema}

Unlike meningioma, VS are rarely associated with significant edema. Rarely, however, there can be significant edema associated in VS [Figure 3]. It is better in such cases to electively do a two-stage microsurgery to achieve total excision with good cranial nerve function.

\section{SS 4: Microsurgery following failed radiosurgery}

We have observed the following in the patients operated by us following failed radiosurgery.

- While more difficult than a virgin case (not more difficult than a redo microsurgery), total excision with good results is possible [Figure 2].

- Tumor is firm but less vascular.

- The arachnoid is usually thicker and adherent to the tumor. In such cases, a thin layer of tumor is better left attached to the brainstem/cranial nerves for better functional outcome.

\section{SS 5: VS in elderly ( $>65$ years)}

- Elderly patients with incidentally picked up tumors or with only longstanding auditory/vestibular

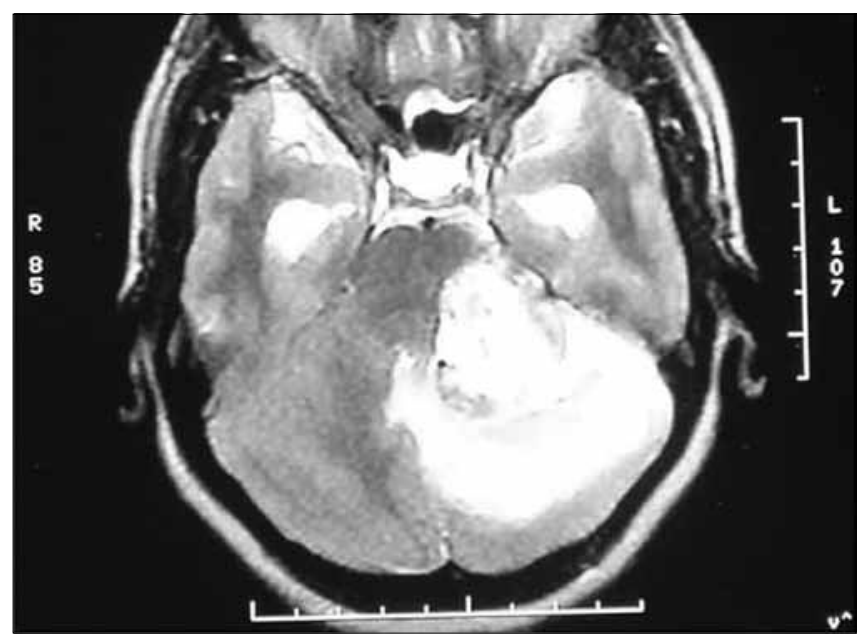

Figure 3: T2W axial MRI showing malignant edema in a case of VS 
dysfunction should be observed for a period of time before considering any treatment.

- Patients with small tumors will generally not need any treatment in their lifetime.

- Patients presenting with normal pressure hydrocephalus (NPH) syndrome are better treated by a ventriculo-peritoneal shunt and generally will not need direct surgery for the tumor.

- While radiosurgery is the preferred modality for elderly patients with tumors less than three $\mathrm{cm}$, patients with larger symptomatic tumors should be operated and age alone should not be a criterion to withhold microsurgery. Dramatic improvement in the quality of life is possible in such patients with microsurgery.

\section{Conclusion}

Vestibular schwannomas of all sizes can be operated microsurgically by the retrosigmoid approach with gratifying results. Both facial function and hearing can be preserved after microsurgery even in large tumors; hence no effort should be spared to save these nerves. There is no doubt, however, that some patients are better served with GKR and yet some others by no intervention at all. Equally important to remember is that microsurgery for VS is a major procedure, there is a steep learning curve and one can lose a patient from a seemingly innocuous few drops of postoperative CSF rhinorrhea.

In summary, the approach for a given patient should be patient-oriented and not surgeon-oriented. Meticulous attention to minute details of each aspect of microsurgery is essential to avoid catastrophe. The indications for GKR and microsurgery are still evolving. Selection of the appropriate option by a surgeon with ample experience, though not easy, is critical to an optimal outcome.

\section{Acknowledgments}

Authors acknowledgment the help of Sarthak B Misra, MBBS, for English language editing and Sudha Nair for secretarial help.

\section{References}

1. Moskowitz N, Long DM. Acoustic neuromas: Historical review of a century of operative series. Neurosurg Quart 1991;1:2-18.

2. Cushing H. Tumours of the nervus acusticus and the syndrome of the cerebellopontine angle. W.B. Saunders Co; 1917. p. 1-295.

3. Pollock BE. Vestibular Schwannoma management: An evidence based comparison of stereotactic radiosurgery and microsurgical resection. Progr Neurosurg 2008;21:222-7.

4. Misra BK, Rout D, Bhiladvala DB. Current status of acoustic neuroma surgery. In: Book of Abstracts. Madras: Neuroconf; 1993.

5. House JW, Brackmann DE. Facial nerve grading system. Otolaryngol Head Neck Surg 1985;93:146-7.

6. Rand RW, Dirks DD, Morgan DE, Bentson JR. Acoustic neuromas. In: Youmans JR, editor. Neurological surgery. 2nd ed. W.B. Saunders Co; 1982. p. 2967-3003.
7. House WF. Monograph 2- acoustic neuroma. Arch Otolaryngol 1968;88:575-715.

8. Shephard RH, Wadia NH. Some observations on atypical features in Acoustic Neuroma. Brain 1956;79:282-318.

9. Dandy WE. Results of removal of acoustic tumours by the unilateral approach. AMA Arch Surg 1941;42:1026-33.

10. Malis LI. Microsurgical treatment of acoustic neuromas. In: Honda H, editor. Microneurosurgery. Baltimore: Univ Park Press; 1975. p. 105-20.

11. Yasargil MG, Fox JL. The microsurgical approach to Acoustic Neuroma. Surg Neurol 1974;2:393-8.

12. Mathies C, Samii M. Vestibular and auditory function: Options in large T3 and T4 tumour. Neurochirurgiere 2002;48:461-70.

13. Prasad D, Steiner M, Steiner L. Gamma Surgery for vestibular schwannoma. J Neurosurg 2000;92:745-59.

14. Flickinger JC, Kondziolka D, Niranjan A, Lunsford LD. Results of acoustic neuroma radiosurgery: An analysis of 5 years experience using current methods. J Neurosurg 2001;94:1-6.

15. Haddad GF, Al-Mefty O. The road less travelled: Trans-temporal access to CPA. Clin Neurosurg 1994;41:150-67.

16. Misra BK, Rout D, Bhiladvala DB, Radhakrishnan V. Spontaneous haemorrhage in Acoustic Neuromas. Br J Neurosurg 1995;9:219.

17. Misra BK. Microsurgical Approach to Cerebello pontine angle tumour. In: 11th ICNS, Monduzzi Editors 1997. p. 363-9.

18. Misra BK. Management of Acoustic Neuroma: An overview in Brain tumour surgery. In: Kanno T, editor. Japan: Brain Tumour Society; 2000. p. 133-8.

19. Misra BK. Changing Trends in the management of acoustic neuroma. Progr Clin Neurosci 2003;18:34-40.

20. Misra BK, Recent trends in the management of acoustic neuroma. In: Khamlichi AE, editor. Medimond: 13th World Congress of Neurological Surgery; 2005. p. 213-20.

21. Misra BK. Modern management protocol and outcome in Acoustic Neuroma. Asian J Neurosurg 2007;1:30-6.

22. Nair S, Rao RM, Kachhara R. Current perspectives in hearing preservation in large acoustic schwannomas. Progr Clin Neurosei 2004;19:216-25.

23. Gabert K, Regis J, Delsanti C, Roche PH, Facon F, Tamura M, et al. Preserving hearing function after gamma knife radiosurgery for unilateral vestibular schwannoma. Neurochirugiere 2004;50:350-7.

24. Lunsford LD, Niranjan A, Flickinger JC, Maitz A, Kondziolka D. Radiosurgery of vestibular schwannoma: Summary of experience in 829 cases. J Neurosurg 2005;102:195-9.

25. Kano H, Kondziolka D, Khan A, Flickinger JC, Lunsford LD. Predictors of hearing preservation after stereotactic radiosurgery for acoustic neuroma. J Neurosurg 2009:Mar 13. [Epub ahead of print].

26. Thomas C, Di Maio S, Ma R, Vollans E, Chu C, Clark B, et al. Hearing Preservation following fractionated stereotactic radiotherapy for vestibular schwannomas: Prognostic implications of cochlear dose. J Neurosurg 2007;107:917-26.

27. Rowe J, Grainger A, Walton L, Sileocks P, Radatz M, Kemeny A. Risk of malignancy after gamma knife stereotactic radiosurgery. Neurosurgery 2007;60:60-6.

28. Niranjan A, Lunsford D, Flickinger JC, Maitz A, Kondziolka D. Can hearing improve after acoustic tumour radiosurgery? Neurosurg Clin N Am 1999;10:305-16.

29. Regis J, Pellet W, Delsanti C, Dufour H, Roche PH, Thomassin JM, et al. Functional outeome after gamma knife surgery or microsurgery for vestibular schwannomas. J. Neurosurg 2002;97:1091-100.

30. Niranjan A, Mathieu D, Flickinger JC, Kondziolka D, Lunsford LD. Hearing preservation after intracanalicular vestibular schwannoma radiosurgery. Neurosurgery 2008;63:1054-62.

31. Coelho DH, Roland JT Jr, Rush SA, Narayana A, St Clair E, Chung W, et al Small vestibular schwannomas with no hearing: comparison of functional outcomes in stereotactic radiosurgery and microsurgery. Laryngoscope 2008;118:1909-16.

32. Raut VV, Walsh RM, Bath AP, Bance ML, Guha A, Tator CH, et al. Conservative management of Vestibular schwannomas - Second review of a prospective longitudinal study. Clin Otolaryngol Allied Sci 2004;29:505-14.

33. Ojemann RG. Management of acoustic neuroma. Clin Neurosurg 1993;40:498-535.

Accepted on 19-06-09

Source of Support: Nil, Conflict of Interest: None declared. 